\title{
PERCEPÇÃO DO PROGRAMA SAÚDE DA FAMÍLIA \\ A PARTIR DE ESTAGIÁRIOS DO PROJETO VER-SUS - VIVÊNCIAS E ESTÁGIOS NA REALIDADE DO SISTEMA ÚNICO DE SAÚDE
}

\author{
PERCEPTIONS OF THE FAMILY HEALTH PROGRAM \\ FROM THE APPRENTICES OF VER-SUS PROJECT; EXPERIENCES AND \\ APPRENTICESHIP IN THE REALITY OF THE PUBLIC HEALTH SYSTEM \\ LA CONCEPCIÓN DEL PROGRAMA SALUD DE LA FAMILIA \\ DE LOS ALUMNOS DE PRACTICA DEL PROYECTO VER-SUS - \\ VIVENCIAS Y PRACTICAS EN EL COTIDIANO DEL SISTEMA ÚNICO DE SAÚDE
}

\author{
Luciana Valadão Vasconcelos Alves* \\ Letícia Silveira Cardoso* \\ Elton D'Avila Dimkoski**
}

* Acadêmicas de Enfermagem e Obstetrícia da Fundação Universidade Federal do Rio Grande - FURG

** Acadêmico de Nutrição da Universidade Regional do Noroeste do Rio Grande do Sul - UNIJUÍ

RESUMO. O presente estudo referiu-se a duas experiências do projeto Vivências e Estágios na Realidade do Sistema Único de Saúde: VER-SUS Rio Grande e Região Noroeste do Rio Grande do Sul. A proposta desse estágio é aproximar os estudantes universitários da área de saúde dos desafios inerentes à implantação do SUS, por meio de vivências em diversas instâncias do sistema de saúde como gestão, atenção básica, educação e controle social. Após a realização destas atividades, os estagiários do VER-SUS relataram suas percepções com o Programa Saúde da Família (PSF), com o objetivo de discutir os aspectos observados durante suas vivências em UBSF, buscando relacioná-los com os princípios e diretrizes do SUS. Para a realização desse estudo foram utilizados os relatórios elaborados pelos estudantes após discussões sobre cada dia de estágio, foi possível identificar questões como estruturas físicas precárias, ausência de medicamentos, filas para o agendamento de consultas, dificuldades para a realização de exames, reconhecimento do Agente Comunitário de Saúde, verticalidade nas relações da equipe, múltiplas formas de atuação das equipes, inexistência de Conselhos Locais de Saúde, denúncias e relatos de usuários e profissionais, além de questões que fomentam a discussão da atuação de profissionais no PSF. Observou-se que a eficácia do PSF depende do contexto em que se encontra.

PALAVRAS-CHAVE: programa saúde da família; sistema único de saúde; saúde pública.

ABSTRACT. The present study came from two experiences of the project "Vivências e Estágios na Realidade do Sistema Único de Saúde: VER-SUS Rio Grande e Região Noroeste do Rio Grande do Sul" (Experiences and Apprenticeship in the Reality of the Public Health System: VER-SUS Rio Grande and Northwest Region of Rio Grande do Sul). The proposal of this apprenticeship is to approach the university students of the health area to the inherent challenges to the implantation of SUS, through experiences in many instances of the system, as management, attention, education and social control. From this on, VER-SUS trainees reported their perceptions on the "Programa de Saúde da Família (PSF)" (Family Health Program) with the objective of discussing the aspects observed during the experiences, trying to relate that with the principles and lines of direction of SUS. For the accomplishment of this study, reports developed by the students, after discussions about each day of apprenticeship, were taken into consideration. From the presented units, it was possible to identify questions as precarious physical structures, lack of medicines, long lines for consultations booking, difficulties for the accomplishment of examinations, the recognition of the Community Health Agent, uprighting in the relations of the team, multiple forms of performance of the teams, non-existence of Local Health Councils, users and professionals' denunciations and reports, besides bringing questions that foment the discussion of the PSF performance. Finally, it was also observed that the effectiveness of the program depends on the context where it is, being the region, the operating team, the managers' support and the users' compromising influential in the production of this.

KEYWORDS: family health program; public health system; public health.

RESUMEN. El presente estudio tuvo como origen, dos experiencias del proyecto "Vivencias y Prácticas en el Cotidiano del Sistema Único de Salud: VER-SUS Rio Grande y Región Noroeste de Rio Grande do Sul. La propuesta de esta práctica, es aproximar los estudiantes universitarios del área de la salud a los desafíos propios de la implementación del SUS, por medio de vivencias en diversas instancias del sistema como gestión, atención, educación y control social. Desde esta experiencia, los alumnos relataron sus percepciones respecto al Programa Salud de la Familia (PSF) con el objetivo de debatir los aspectos observados durante sus vivencias, y, de esta forma, buscando relacionar con los principios y directrices del SUS. Para la realización de este estudio, se utilizó los apuntes elaborados por los estudiantes tras los debates sobre cada día de práctica. De las unidades presentadas, fue posible identificar cuestiones como estructuras físicas precarias, falta de medicinas, filas para marcar una consulta médica, dificultades para la realización de los exámenes clínicos, el reconocimiento del Agente Comunitario de Salud, rigidez en las relaciones del equipo, múltiples formas de actuación del equipo, inexistencia de los Consejos Locales de Salud, denuncias y relatos de usuarios y profesionales, además de presentar cuestiones que fomentan la discusión de la actuación del PSF. Por último, se observó que la eficacia del programa depende del contexto en que se encuentra

PALABRAS-CLAVE: programa de salud de la familia; sistema único de salud; salud publica.

Recebido em: 09/03/2005

Aceito em: $\quad 07 / 06 / 2005$
Luciana Valadão Vasconcelos Alves

Rua dos Âncoras, 211 - Bairro Parque Marinha - 96215-560

E-mail: lucianavaladao@ibest.com.br 


\section{INTRODUÇÃO}

"O Projeto de Vivências e Estágios na Realidade do Sistema Único de Saúde - VERSUS/Brasil faz parte da estratégia do Ministério da Saúde e do Movimento Estudantil da área da saúde para aproximar estudantes universitários do setor aos desafios inerentes à implantação do Sistema Único de Saúde (SUS). A meta é contribuir para a formação de profissionais críticos e sensíveis às necessidades da população brasileira e implementar o fortalecimento do SUS. Além disso, espera-se a criação de novas relações de compromisso e de cooperação entre estudantes, gestores de saúde, instituições de ensino superior e movimentos sociais, para efetivar a integralidade em saúde e a educação adequada dos profissionais. Durante a participação no projeto VERSUS, os protagonistas têm a oportunidade de vivenciar conquistas e desafios inerentes ao SUS. Podem também aprofundar a discussão sobre o trabalho em equipe, gestão dos serviços, atenção, educação e controle social no sistema" 1:5.

A construção do projeto VER-SUS partiu da necessidade de acadêmicos de saúde de conhecer/ entender o modelo atual de saúde no Brasil - o SUS, tendo como objetivos valorizar e potenciar o compromisso ético-político dos participantes no processo de implantação do SUS, provocar reflexões acerca do papel do estudante como agente transformador da realidade social, colaborar no amadurecimento da prática multiprofissional e interdisciplinar e contribuir para o debate sobre o projeto político-pedagógico da graduação em saúde e sobre a implementação das diretrizes curriculares nacionais e das diretrizes constitucionais do SUS, fortalecendo os compromissos do SUS com o ensino da saúde.

\section{METODOLOGIA}

Os relatos feitos a seguir foram fruto de duas vivências: VER-SUS Rio Grande e VER-SUS Região Noroeste, ambos no Rio Grande do Sul (RS). O estágio na cidade do Rio Grande ocorreu em julho de 2005, e contou com a participação de 30 acadêmicos dos cursos de enfermagem, biologia, psicologia, farmácia, odontologia, fisioterapia, nutrição e serviço social, provenientes de diversas universidades do estado. O projeto VER-SUS Região Noroeste do RS, com sede em ljuí, ensejou vivências também nas cidades de Santa Rosa, Tenente Portela, Alecrim, Porto Mauá, Três de Maio e Catuípe. O estágio ocorreu em janeiro de 2006, com a participação de 24 estudantes, sempre contemplando a multidisciplinaridade. No VER-SUS Região Noroeste houve a participação de acadêmicos do curso de medicina. Foi realizado intercâmbio dos estudantes que atuaram nas cidades em que foi realizado o projeto.

Durante os 15 dias de vivência, os acadêmicos conheceram diferentes estâncias onde o SUS é implementado, como unidades básicas de saúde, unidades básicas de saúde da família, hospitais, conselhos municipais de saúde, centro de atenção psicossocial (CAPS) e secretarias de saúde, além de comunidades locais, movimentos sociais e universidades. Após cada dia de estágio, os participantes debatiam as situações vistas e elaboravam relatórios, tendo como base para as discussões o uso de textos sobre o assunto, palestras assistidas durante o processo e apoio de facilitadores, geralmente ex-estagiários do VER-SUS.

Os relatórios foram feitos diariamente por um grupo de estagiários que procuravam expor as percepções de cada local visitado, também acrescentavam relatos de usuários e profissionais de saúde.

Com essa experiência foi possível observar diferentes formas de implementação do Programa Saúde da Família, realizadas conforme a região em que se insere e a comunidade que assiste. A partir da realidade encontrada, resolveu-se discutir o PSF destes locais, tentando analisá-lo tendo como base a revisão da literatura referente aos princípios e às diretrizes do SUS e do PSF.

"Para a reorganização da atenção básica no país, o Ministério da Saúde criou em 1994 o Programa Saúde da Família (PSF) cuja estratégia incorpora e reafirma os princípios básicos do Sistema Único de 
Saúde (SUS) - universalidade, integralidade, eqüidade, descentralização e participação social" ${ }^{2}$. Com o objetivo de levar a saúde para mais perto da família, sua estrutura está inserta na comunidade e organizada a partir da Unidade Básica de Saúde da Família (UBSF). Segundo o Ministério da Saúde, o programa, "ao contrário do modelo tradicional, centrado na doença e no hospital, prioriza as ações de proteção e promoção à saúde dos indivíduos e da família, tanto adultos, quanto crianças, sadios ou doentes, de forma integral e contínua" ${ }^{3}$.

"A equipe multiprofissional é composta, no mínimo, por um médico, um enfermeiro, um técnico de enfermagem e de quatro a seis agentes comunitários de saúde (ACS). Outros profissionais, como dentistas, assistentes sociais e psicólogos, podem ser incorporados às equipes ou formar equipes de apoio, de acordo com as necessidades e possibilidades locais. Esses profissionais se responsabilizam pela situação de saúde de determinada área, cuja população deve ser no mínimo de 2.400 e no máximo 4.500 pessoas. A população deve ser cadastrada e acompanhada, tornando-se responsabilidade das equipes atendê-la, entendendo suas necessidades de saúde como resultado também das condições sociais, ambientais e econômicas em que vive" ${ }^{4(41)}$. O diferencial do PSF é o atendimento prestado tanto na unidade de saúde como em domicílio, assim facilitando o "objetivo principal desse programa, que é justamente aproximar as equipes da comunidade e estabelecer entre elas vínculos sólidos" 4:41.

\section{OBJETIVO}

Discutir os aspectos observados no PSF durante as vivências do VER-SUS Rio Grande e Região Noroeste do Rio Grande do Sul, buscando relacionar com os princípios e diretrizes do SUS.

\section{RESULTADO}

Em Rio Grande foram visitadas nove (9) Unidades Básicas de Saúde da Família (UBSF). Na região Noroeste do estado foram visitadas em ljuí quatro (4) unidades com PSF, em Santa Rosa (2) duas em Três de Maio, (1) uma UBSF, nesta região há dez (10) UBSF. As demais cidades do VER-SUS Região Noroeste do Rio Grande do Sul ainda não haviam implementado o PSF.

A partir das vivências e estágios realizados, observou-se vários aspectos relevantes no processo de trabalho desenvolvidos nas UBSF, como estruturas físicas precárias, ausência de medicamentos, filas para o agendamento de consultas, dificuldades para a realização de exames, reconhecimento do ACS, verticalidade nas relações da equipe entre outros. Após discussões e análises dos dados obtidos, os grupos de estudantes construíram relatórios parciais, denotando os aspectos positivos e negativos dos locais. Assim, para delimitar as prioridades do PSF, consideram-se os pontos mais abrangentes de todos os relatos.

Inicialmente, observou-se na cidade do Rio Grande que algumas unidades são casas alugadas e não apresentam estrutura física adequada, pois o ambiente é úmido, frio, pequeno e com esgoto a céu aberto, não possibilitando atendimento qualificado à população. Em contrapartida foram encontradas unidades com boa infra-estrutura, atendendo as necessidades locais. Foi verificada, também a falta de medicamentos e seu armazenamento precário em grande parte dos locais visitados. Em ljuí podese notar melhor estrutura física na maioria das unidades, embora algumas possuam ambiente consideravelmente pequeno pelo número de habitantes que atende. Em Santa Rosa vê-se UBSF com estruturas físicas planejadas para possibilitar total interação dos profissionais, pois os consultórios são próximos e localizados num único corredor separado da recepção. Nas cidades da região Noroeste percebeu-se o mesmo problema que em Rio Grande, quanto ao inadequado armazenamento dos medicamentos, como exposição a luz solar e umidade. Contudo essa população pouco relatou a falta de medicamentos básicos nas unidades, o que evidencia a eficácia desse setor.

Outra questão relevante são as filas formadas para o agendamento das consultas na unidade, os 
usuários vão para a fila no início da noite anterior para conseguir uma consulta para o dia seguinte, permanecendo na fila por um período de mais ou menos dez horas, Isso é resultante das poucas fichas disponibilizadas para a demanda. Embora, parte das pessoas que procuram o serviço sejam atendidas, devido às fichas distribuídas diariamente, torna-se necessário a ampliação dessas consultas para abranger toda a comunidade.

Os usuários relataram a dificuldade para realizar os exames solicitados pelos médicos do PSF; além de terem que se deslocar várias vezes do seu bairro, enfrentam filas e aguardam até meses para sua realização. Isso evidencia que o sistema referência e contra-referência não funciona adequadamente, deixando déficits no atendimento do paciente. Como exemplo de algumas unidades, são feitas coletas de amostras para a realização de exames de fezes e urina, onde se sugere a implementação de tal atividade em todos locais do PSF de Rio Grande, o que evitaria que o usuário tivesse que se deslocar de seu bairro até o centro da cidade para realizar tais procedimentos. Além disso, a demora para o atendimento da ambulância foi observada em muitos locais visitados das regiões. Seria interessante um atendimento mais ágil das ambulâncias disponibilizadas e distribuí-las de forma estratégica em áreas do PSF ou próximo.

Percebeu-se, nos relatos dos usuários e dos Agentes Comunitários de Saúde (ACS), que a comunidade aceitou muito bem a implantação do programa. Em ljuí alguns ACS relataram que sua função é de prevenção e monitoramento das condições de saúde das famílias cadastradas e percebem durante as visitas uma boa aceitação por parte da comunidade. Durante o estágio foi possível acompanhar os ACS nas visitas domiciliares; observou-se que esse profissional demonstra conhecimento das necessidades das pessoas visitadas, uma facilidade de diálogo e comprometimento com os indivíduos, realizando pactos de colaboração para desenvolverem ações resolutivas dos problemas apresentados por eles. Além de possuírem uma interação mais próxima do ambiente familiar, o ACS é um dos meios de informação do usuário e o elo de ligação entre a equipe e a comunidade, assim, salienta-se que é necessária uma capacitação mais efetiva em termos de proporcionar maiores conhecimentos a esses profissionais. É interessante acrescentar uma experiência observada pelos estagiários, em ljuí, sobre um grupo de ACS que produziam um sabonete medicinal utilizado no combate da escabiose e da pediculose, que era doado à população num mutirão da saúde.

Paralelamente, em alguns serviços, foi observada a verticalização nas relações entre os membros da equipe, demonstrando a pouca integração dos seus profissionais, verticalidade nas relações destes. Observou-se baixa transdisciplinaridade no processo de trabalho das equipes multiprofissionais do PSF, uma vez que, não existe troca de informações suficiente entre os trabalhadores nos diversos setores de atuação: prevenção, promoção, reabilitação.

Na vivência da região Noroeste do Rio Grande do Sul o grupo enfatizou, em vários momentos, a questão da saúde do trabalhador do PSF. Ela foi apresentada como necessidade de cuidador do trabalhador atuante nas unidades de saúde através de apoio psicológico; eles relatam sentir a angústia, devido à sua atuação no sistema; alguns profissionais referem sentirem-se frustrados devido a que o trabalho não alcança os resultados esperados ou suas atividades se tornam limitadas por ausência de recursos financeiros, estruturais entre outras dificuldades encontradas pelas equipes do PSF. Além disso, notou-se que o trabalho desenvolvido em comunidades carentes sensilibiza o profissional, o que muitas vezes afeta seu estado emocional; ao mesmo tempo ele procura não realizar atividades assistencialistas; ele depara com situações de miséria que o estimulam a questionar os limites entre atuação profissional e atuação cidadã e solidária.

Concomitantemente, observou-se que um dos fatores agravantes à saúde são as condições higiênico-sanitárias das comunidades, o que impossibilita a realização plena do trabalho das 
equipes, uma vez que as pessoas adoecem, são tratadas, mas continuam com suas práticas/costumes, situação essa que possivelmente favorece a descrença no trabalho comunitário, gerando as angústias profissionais citadas anteriormente.

Outra questão a ser destacada é a inexistência de Conselhos Locais de Saúde (CLS) nas cidades visitadas durante o VER-SUS. Sabe-se que é um direito e um dever da sociedade participar das gestões públicas em geral e da saúde pública em particular, reconhecendo-se que, a partir do envolvimento da sociedade na construção e fiscalização do SUS, este terá maiores chances de êxito. Sugere-se, portanto, a formação de CLS, a fim de que a população participe, junto com profissionais de saúde da busca, de soluções para melhorar sua qualidade de vida.

Notou-se também a presteza da implementação da equipe de saúde bucal, que já possui espaço físico em algumas unidades de saúde. Em vista de que, em uma das localidades visitadas no VER-SUS Rio Grande, foi apresentada uma equipe de odontólogos bem organizada e dedicada. Nesta, o profissional leva cerca de meia hora no atendimento a cada paciente, evidenciando a preocupação e o cuidado com o dever. Paralelamente, há assistência a toda a família com prevenção e orientação aos pacientes, a fim de evitar a extração dos dentes desnecessariamente. Embora a equipe não consiga atender à demanda, mostra-se interessada no atendimento que presta. Além disso, realizam palestras de prevenção nas creches e aplicação de flúor nas crianças, às vezes até em casa. Em contrapartida, verificou-se que a demanda pelos serviços de nutrição e psicologia é muito alta, devido ao baixo número desses profissionais na rede municipal de Rio Grande e Região Noroeste.

Foi observada nas duas regiões a ação dos grupos educativos direcionados a hipertensos, diabéticos, adolescentes, planejamento familiar, implantadas em diversas unidades de saúde. Eles conseguem informar e aproximar o usuário dos profissionais de saúde. Acreditamos ser interessante a realização destas atividades nos locais onde ainda não é executada, estimulando a criação de grupos de saúde mental, trabalhados em poucas UBSF destes locais.

\section{CONCLUSÃO}

Ao desenvolver o estágio do VER-SUS, percebemos que a UBSF é um espaço que os acadêmicos possuem para se aproximar da comunidade; ao acompanhar os ACS nas visitas domiciliares, facilita-se essa aproximação e proporciona interação com as famílias e novas descobertas. Acreditando que, a partir dessa interação, seria interessante trabalhar com o usuário, tentando integrar o conhecimento científico ao conhecimento popular para promover a saúde, a educação e a transformação social. Enfim, o PSF proporciona infinitas estratégias de trabalho conjunto com a comunidade, mas é importante a organização e dedicação da equipe para tais realizações, visto que se notaram experiências durante esta pesquisa no VER-SUS que é difícil mobilizar a população para essas ações.

"Seguindo o princípio da integralidade, as atividades de educação em saúde estão incluídas entre as responsabilidades dos profissionais do PSF" 7 , sendo interessante ressaltar que nem todas unidades visitadas trabalhavam esse assunto.

Sem dúvida, é necessária nova formação acadêmica, em que as as instituições de Ensino Superior devem proporcionar, desde o início dos cursos da área da saúde, disciplinas de Educação em Saúde Coletiva, promovendo debates e reflexões práticas, conhecimentos e ação de saúde da população ${ }^{1}$. Além disso, é preciso formar profissionais comprometidos com a comunidade e estimulados à mobilização social, que busquem a participação efetiva da população, onde todos sejam co-participantes e responsáveis em assim é possível concretizar os objetivos do PSF.

Ficou evidente situações que não contemplam os princípios e diretrizes do SUS, como a inexistência dos Conselhos Locais de Saúde indo contra a diretriz 
que aborda o Controle Social (participação da comunidade) como "responsabilidade e direito do usuário" 5 . Outra questão é o direito ao "atendimento integral, com prioridade para as atividades preventivas, sem prejuízo dos serviços assistenciais" ${ }^{6}$, ou seja, além da prevenção e da promoção de saúde, o usuário tem direito aos serviços assistenciais tanto de tratamento como de reabilitação. Porém foi observado, durante o estágio, que existem medicações insuficientes nas unidades, o que impossibilita o acompanhamento ambulatorial de casos como hipertensão, diabetes e outras enfermidades.

Percebeu-se que parte de alguns profissionais tem dificuldade de ver o usuários como ser biopsicossocialcultural, de observar às características e necessidades, relacionando-as ao características do meio onde ele está inserto, sendo, então, os atendimentos e tratamentos voltados aos sintomas fisiológicos. Exemplo disso é a dificuldade de trabalhar com saúde mental. Isso pode ser revertido com uma capacitação mais generalista do profissional que atua no PSF, fazendo com que ele se sinta capaz de suprir as necessidades da população. O ACS é o profissional que mais demonstrou preocupação com os determinantes psicossociais e culturais da população, apontando questões como comportamentos orientados por crenças, valores e saberes, além do meio social em que o indivíduo está inserto como influência à adesão das práticas de saúde.

Uma vez que a equipe do programa é multiprofissional, com no mínimo médico, enfermeiro, técnico de enfermagem e ACS, esses devem trabalhar em conjunto, trocando saberes/conhecimentos, como prioriza a interdisciplinaridade, em prol de melhor assistência da comunidade. Para isso é necessária uma afirmação dos profissionais do PSF quanto a eficácia do trabalho inter e multidisciplinar para uma compreensão mais abrangente dos problemas de saúde e intervenções mais efetivas ${ }^{7}$.

Como se observa nas vivências e afirmado por TRAD e BASTOS, "o investimento em educação continuada e permanente da equipe de saúde e em informação em saúde destinada à população tem surtido efeitos positivos no que se refere à qualidade da prática assistencial e à adoção de hábitos mais saudáveis por parte da população; por exemplo através dos grupos educativos vistos" ${ }^{8}$. Com isso, nota-se a importância de realizar capacitações freqüentes dos profissionais da equipe, pois é por meio desses que a comunidade recebe orientações, tanto nas consultas quanto nos grupos de apoio.

Conclui-se, após analisar os aspectos mencionados, que o PSF está mudando a relação usuário-profissional. $\mathrm{O}$ contato feito com a comunidade é o diferencial que proporciona um atendimento mais próximo possível da realidade dos usuários. Embora muitos aspectos negativos tenham sido observados, fica evidente que o Programa está em construção e que as mudanças propostas requerem tempo. A partir da vivência, percebeu-se que os usuários estão aceitando progressivamente o PSF, visto que esta aceitação tende a se ampliar e afirmar conforme os déficits sejam trabalhados.

\section{REFERÊNCIAS}

1 Ministério da Saúde (BR). Secretária de gestão do trabalho e da educação na saúde, departamento de gestão da educação em saúde. VER-SUS/Brasil: cadernos de textos. - Brasília: Ministério da Saúde; 2004.

2 Ministério da Saúde (BR). Departamento de atenção básica. Disponível em: <http://dtr2004.saude.gov.br/dab/ conhecadab.php>. Brasília(DF); 2006.

3 Ministério da Saúde (BR). Saúde dentro de casa. Programa de saúde da família. Brasília: Fundação Nacional de Saúde; 1994.

4 Ministério da Saúde (BR). Secretaria Executiva. Departamento de Apoio à Descentralização. O SUS no seu município: garantindo saúde para todos. Brasília: Ministério da Saúde; 2004.

5 Lei Federal no 8080 que institui o Sistema Único de Saúde (SUS) - De 19 de setembro de 1990 - Brasil. Em especial o Capítulo II.

6 Constituição da República Federativa do Brasil. Capítulo II, Seção II da Saúde, artigo 198. Brasil; 1988.

7 Alves VS. Um modelo de educação em saúde para o Programa Saúde da Família: pela integralidade da atenção e reorientação do modelo assistencial. Interface 2005; 9(16):39-52.

8 Trad LAB, Bastos ACS. O impacto sociocultural do Programa de Saúde da Família (PSF): uma proposta de avaliação. Cad. Saúde Pública 1998; 14(2):429-35. 